\title{
Reduction Clitoroplasty with Preservation of the Dorsal Neurovascular Pedicle in a 6-Year-Old Female: A Case Report
}

\author{
Hyochun Park, Wonwoo Kim, \\ Hoonnam Kim, Hyeonjung Yeo \\ Department of Plastic and Reconstructive \\ Surgery, Daegu Fatima Hospital, Daegu, \\ Korea
}

No potential conflict of interest relevant to this article was reported.

\begin{abstract}
A 6-year-old female presented to our hospital with clitoromegaly. On physical examination, she demonstrated evidence of precocious pubic hair and clitoromegaly. The relaxed clitoris measured $3.7 \mathrm{~cm}$ in length. Her uterus was undetectable on diagnostic transvaginal ultrasonography and abdomen-pelvis computed tomography (CT) with otherwise normal-appearing internal female genitalia. We planned reduction clitoroplasty with preservation of the dorsal neurovascular pedicle to maintain sensitivity of the glans clitoris. After surgery, the patient demonstrated good recovery. The parents of the patient were satisfied with the surgical results. The patient was discharged without complications on hospital day 6.
\end{abstract}

Keywords Adrenal hyperplasia congenital, Clitoris, Clitoromegaly

\section{INTRODUCTION}

In adults, the average width of the clitoris is usually 3 to $4 \mathrm{~mm}$, and the length is 4 to $5 \mathrm{~mm}$ [1]. The most common cause of clitoral hypertrophy in the newborn is congenital adrenal hyperplasia [2]. Twenty-one-hydroxylase $(\mathrm{OH})$ deficiency accounts for about $90 \%$ of cases. The incidence is 1:5,000 to 1:15,000 live births and is characterized by reduced production of cortisol and aldosterone and increased 17-OH-progesterone (OHP) and sex steroids. The excess androgens cause virilization of the female and ambiguous genitalia. This case report describes a 6-year-old female with congenital adrenal hyperplasia. She underwent a clitoral reduction procedure in which the corpora cavernosa were removed, while the dorsal neurovascular bundle was preserved.

Received: Dec 9, 2015 Revised: Mar 4, 2016 Accepted: Mar 21, 2016 Correspondence: Hyochun Park Department of Plastic and Reconstructive Surgery, Daegu Fatima Hospital, 99 Ayang-ro, Dong-gu, Daegu 41199, Korea. E-mail: phc3900@hanmail.net

Copyright @ 2016 The Korean Society for Aesthetic Plastic Surgery.

This is an Open Access article distributed under the terms of the Creative Commons Attribution Non-Commercial License (http://creativecommons.org/licenses/by-nc/4.0/) which permits unrestricted non-commercial use, distribution, and reproduction in any medium, provided the original work is properly cited. www.e-aaps.org

\section{CASE REPORT}

A 6-year-old female presented to our hospital with clitoromegaly. The pediatric department diagnosed the patient with 21-OH deficiency, and she was treated with oral hydrocortisone. There were no complications. On physical examination, she had ambiguous genitalia, precocious pubic hair, and clitoromegaly (Fig. 1). The relaxed clitoris measured $3.7 \mathrm{~cm}$ in length. She had grown $16 \mathrm{~cm}$ in height over the previous year. Her diagnostic transvaginal ultrasound and abdominal and pelvic computed tomography (CT) showed an invisible uterus with otherwise normal-appearing internal female genitalia. The patient's karyotype was 46XX. Serum 17-OHP and testosterone levels were increased. We planned reduction clitoroplasty with preservation of the dorsal neurovascular pedicle to maintain the sensitivity of the glans clitoris. First, a 360-degree circumferential incision line $1 \mathrm{~cm}$ proximal to the glans sulcus was designed to cover the glans clitoris with the inner mucosa of the prepuce, and a ventral midline incision line was designed (Fig. 2). Next, a stay suture was fixed on the glans. After making an incision along the lines, the corpora cavernosa were dissected from the glans sulcus to the symphysis pubis. Two longitudinal incisions having a wide interval were designed on the Buck's fascia to include the dorsal neurovascular bundle. The dorsal neurovascular bundle with Buck's fascia and a strip of tunica albuginea was separated from the 


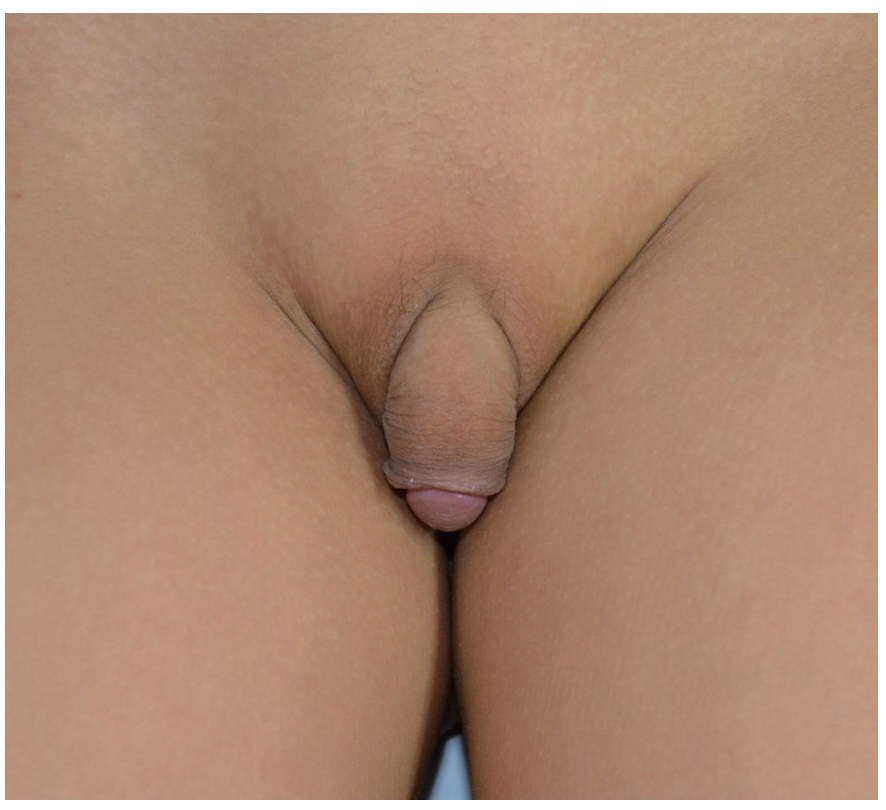

Fig. 1. Preoperative photo showing ambiguous genitalia, precocious pubic hair, and clitoromegaly.

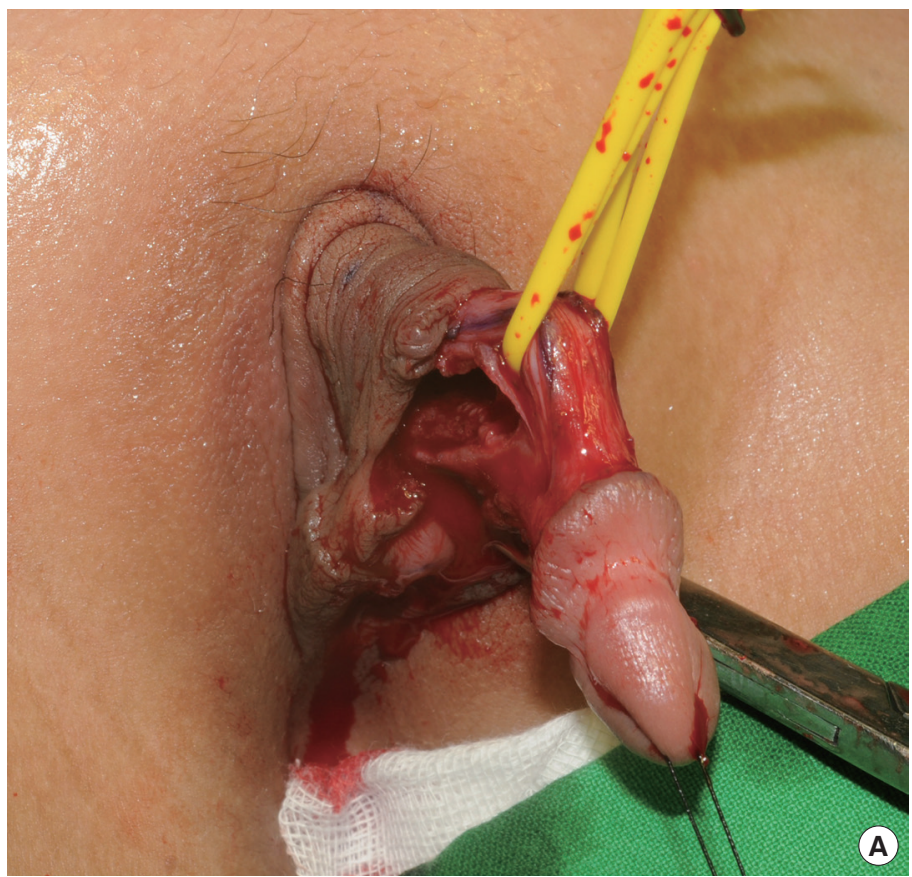

Fig. 3. (A) The neurovascular bundle and corpora cavernosa were separated by a vessel loop. (B) Incision line for glans reduction on the ventral side.

corpora cavernosa (Fig. 3A). After that, the corpora cavernosa were completely removed, from the symphysis pubis to the glans sulcus. To reduce the glans size, an incision line was designed on the ventral side of the glans, and reduction of the glans was performed (Fig. 3B). Next, after folding down the dorsal neurovascular bundle, the base of the glans was sutured to the remnant corpora on the sym-
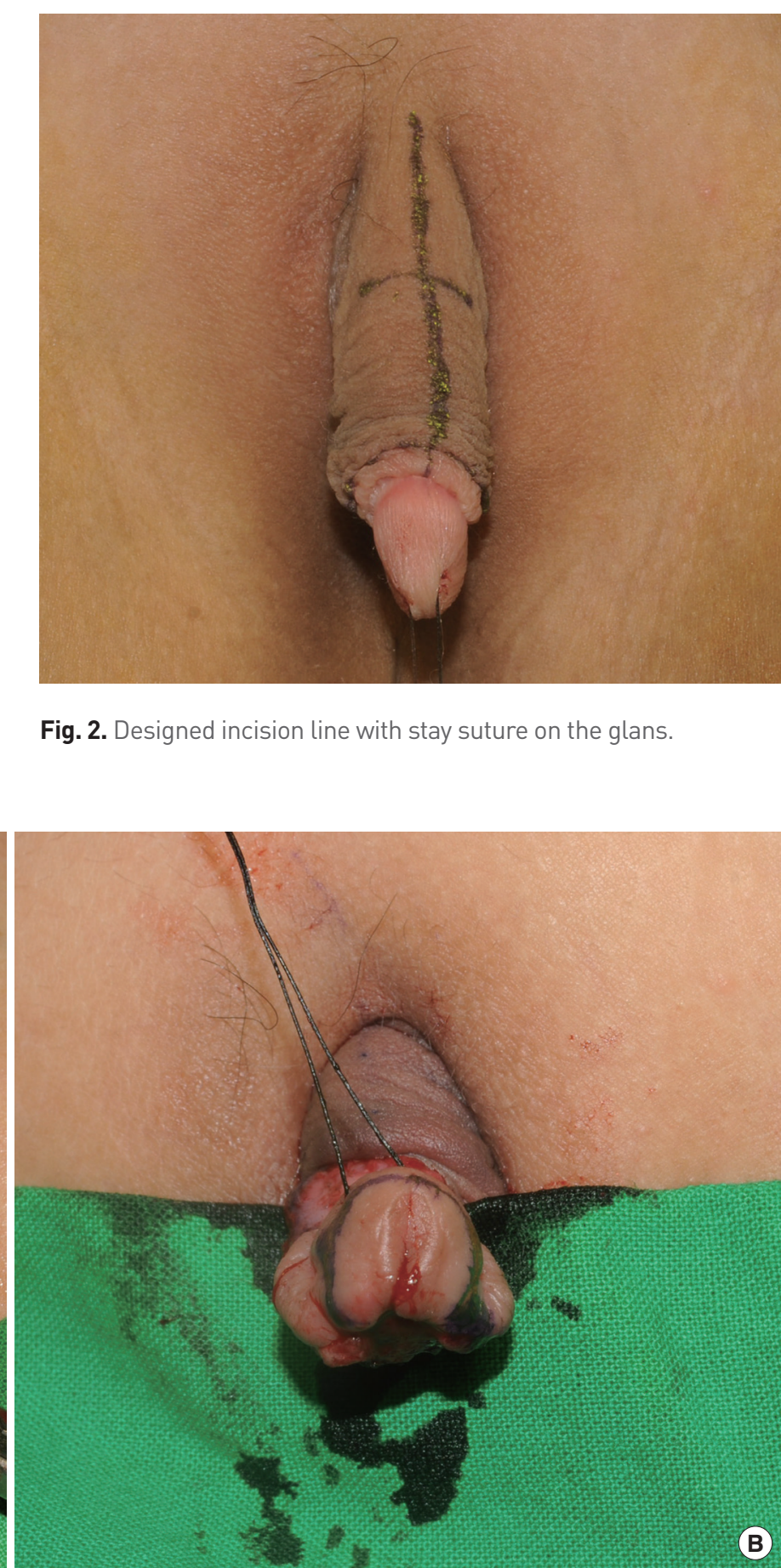

Fig. 2. Designed incision line with stay suture on the glans. 


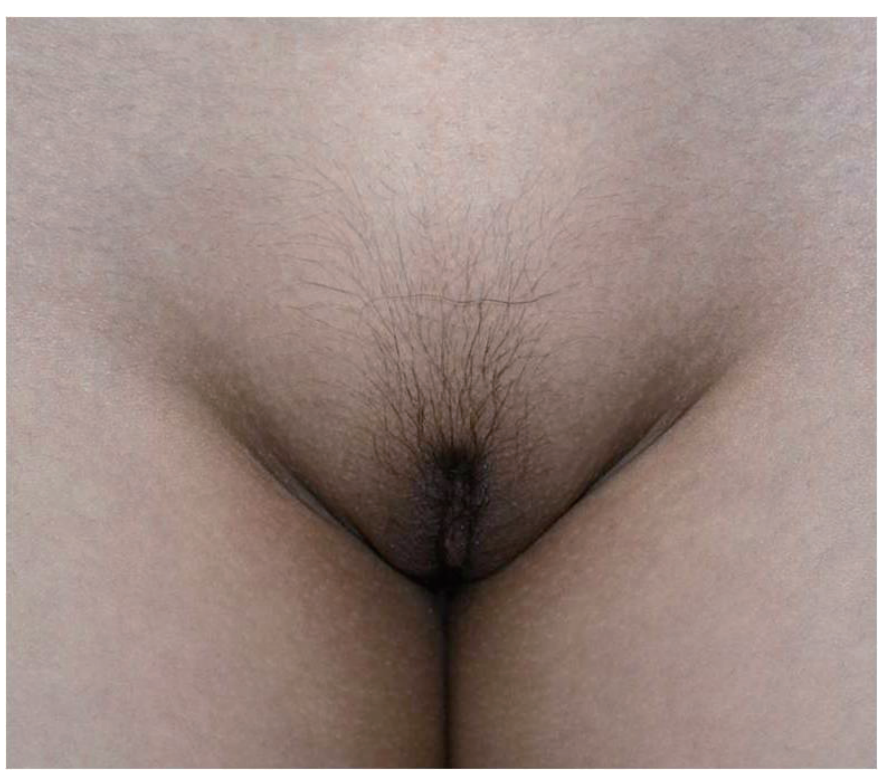

Fig. 4. Postoperative appearance 13 months after surgery.

\section{DISCUSSION}

Causes of clitoromegaly are divided into hormonal factors such as congenital adrenal hyperplasia and non-hormonal factors such as tumor. Other etiologies include idiopathic causes and pseudoclitoromegaly [3]. Clitoromegaly causes serious psychological stress, and treatment should be performed before 6 to 12 months of age in order to minimize any psychological sequelae [4]. A careful history, physical examination, and hormonal analysis are needed to reach an underlying cause, because clitoromegaly may result from a variety of factors [5]. Techniques for clitoral resection have changed a great deal over the past thirty years as our knowledge of the anatomy has improved. In the past, removal of the whole clitoris (clitorectomy) was used, but is not now an acceptable option because the clitoris is recognized as an important sensory organ involved in sexual response [6]. Later, surgeons tried to preserve as much of the enlarged clitoris as possible and buried the corpora under the skin. However, this meant that the erectile tissue was concertinaed under the skin and, when erect, was very painful [7]. Further modifications of the technique involved excision of corporal tissue and preservation of the glans and the neurovascular bundle to reduce any loss of sensation [8]. On the basis of an extensive study of these techniques, we individualized and combined the most important points of other techniques with our refinements to obtain a better cosmetic result and to preserve clitoral sensitivity. For example, in Papageorgiou et al. [4], both the dorsal and ventral neurovascular bundles were preserved and the glans was untouched. In Kogan et al. [9], the dorsal neurovascular bundle was preserved and the dorsal central wedge glans reduction was performed. However, the surgeon did not reduce the size of the glans clitoris sufficiently, which resulted in an unsightly longitudinal scar on the dorsum of the glans clitoris. Selvaggi et al. [8] removed the glans clitoris bilaterally, but we reduced the glans on the ventral side where the density of nerves is lowest [10]. Ventral glans reduction also has the advantage of hiding the surgical scar and easing the removal of a sufficient amount of tissue. Additionally, we left a wide width of the dorsal neurovascular bundle. This is due to the dorsal nerve, which is widely distributed on the dorsum of the phallus. Finally, in order to cover the glans in a resting state, we constructed a prepuce by trimming the skin. By modifying the various procedures used previously, this operation satisfied both the functional and aesthetic desires of the patient and patient's parents.

\section{PATIENT CONSENT}

Patients provided written consent for the use of their images.

\section{REFERENCES}

1. Verkauf BS, Von Thron J, O’Brien WF. Clitoral size in normal women. Obstet Gynecol 1992;80:41-4.

2. Committee on Genetics, Section on Endocrinology, Section on Urology. Evaluation of the newborn with developmental anomalies of the external genitalia. American Academy of Pediatrics. Committee on Genetics. Pediatrics 2000;106:138-42.

3. Copcu E, Aktas A, Sivrioglu N, et al. Idiopathic isolated clitoromegaly: a report of two cases. Reprod Health 2004;1:4.

4. Papageorgiou T, Hearns-Stokes R, Peppas D, et al. Clitoroplasty with preservation of neurovascular pedicles. Obstet Gynecol 2000;96:821-3.

5. Linck D, Hayes MF. Clitoral cyst as a cause of ambiguous genitalia. Obstet Gynecol 2002;99:963-6.

6. Mahmud G, Abbas S. Reduction clitoroplasty for clitoromegaly. Ann Pak Inst Med Sci 2008;4:174-5.

7. Schober JM. Long-term outcomes and changing attitudes to intersexuality. BJU Int 1999;83 Suppl 3:39-50.

8. Selvaggi G, Ressa CM, Ostuni G, et al. Reduction of the hypertrophied clitoris: surgical refinements of the old techniques. Plast Reconstr Surg 2008;121:358e-61e.

9. Kogan SJ, Smey P, Levitt SB. Subtunical total reduction clitoroplasty: a safe modification of existing techniques. J Urol 1983;130:746-8.

10. Baskin LS, Erol A, Li YW, et al. Anatomical studies of the human clitoris. J Urol 1999;162:1015-20. 